\title{
MONOTONIC PROPERTIES OF THE GREEN'S FUNCTION
}

KENNETH S. MILLER AND MENAHEM M. SCHIFFER

1. Introduction. In a previous paper $^{1}$ we obtained variational formulae for the Green's function of an ordinary differential system under the assumption that the interval, operator, and boundary conditions were allowed to vary. We shall now deduce various monotonous properties of the Green's function as well as other results based on these formulae.

Our interest is centered on a linear differential operator

$$
L=p_{0}(x) \frac{d^{n}}{d x^{n}}+p_{1}(x) \frac{d^{n-1}}{d x^{n-1}}+\cdots+p_{n}(x) .
$$

whose coefficients are continuous in some closed finite interval $I$ of the $x$-axis and $p_{0}(x)>0$ in $I$. With Equation (1) we shall associate two-point boundary conditions of the form

$$
U_{\alpha}(u)=\sum_{i=1}^{n} c_{\alpha, i} u^{(i-1)}(a)+\sum_{i=1}^{n} c_{\alpha, n+i} u^{(i-1)}(b), \quad i=1,2, \cdots, n,
$$

where $[a, b]$ is a closed subinterval of $I$. The completely homogeneous system

$$
L u=0, \quad U_{\alpha}(u)=0, \quad \alpha=1,2, \cdots, n,
$$

will always be assumed incompatible. The adjoint of $L$ will be denoted by $L^{+}$and the adjoint boundary conditions by $U_{\alpha}^{*}(u)$. If $L$ or $U$ operates on a function of more than one variable, a presuperscript, for example ${ }^{\zeta} L$ or ${ }^{5} U$, will denote which variable is to be operated upon. The bilinear concomitant will be denoted by $\pi[u, v]$,

$$
\int_{a}^{b}\left[v(x) L u(x)-{ }^{a} u(x) L^{+v}(x)\right] d x=\pi[u, v] .
$$

For every incompatible differential system of the form of Equation (3) there exists a Green's function $G(x, \xi)$. It may be that this Green's function depends on a parameter $\lambda$. Then we shall write

Presented to the International Congress of Mathematicians, September 6, 1950; received by the editors February 27, 1952.

1 On the Green's function of ordinary differential systems, Proceedings of the American Mathematical Society vol. 3 (1952) pp. 433-441. We shall refer to this paper as [MS]. 
$G(x, \xi)=G(x, \xi, \lambda)$ to exhibit this dependence explicitly. If we now consider the same system when $\lambda$ has been changed to $\lambda^{\prime}$, we shall write $G^{\prime}(x, \xi)=G^{\prime}\left(x, \xi, \lambda^{\prime}\right)$ as the Green's function of the new system. We define $\Delta G$ by the equation

$$
\Delta G(x, \xi)=G^{\prime}\left(x, \xi, \lambda^{\prime}\right)-G(x, \xi, \lambda)
$$

which also equals

$$
\Delta G(x, \xi)=\frac{\partial}{\partial \lambda} G(x, \xi, \lambda) \Delta \lambda+O\left(\Delta \lambda^{2}\right) .
$$

Let $\delta G(x, \xi)$ be the principal part of the above expression, that is,

$$
\delta G(x, \xi)=\frac{\partial}{\partial \lambda} G(x, \xi, \lambda) \delta \lambda
$$

where the finite increment $\Delta \lambda$ of the independent variable $\lambda$ has been identified with $\delta \lambda$. Equation (5) is called a finite comparison formula and Equation (7) an infinitesimal variational formula.

We shall consistently use the notation of [MS] in the following sections. In particular, it will frequently be convenient to use the notation $\partial^{r} G(x, b) / \partial 2^{r}$ to indicate that the function $G(x, t)$ was first differentiated with respect to the second argument, $t$, and this argument then replaced by $b$.

\section{Monotonic properties of the Green's function.}

2.1 Variation of the interval. One of the main advantages in the theory of partial differential equations of having variational formulae for the Green's function is that it enables one to deduce many monotonous properties of the Green's function. This is also true in the case of ordinary differential systems. In particular, we shall assume that the differential operator $L$ under consideration is of even order $n=2 r$ and the boundary conditions are of the special form

$$
U_{\alpha}(u)=u^{(\alpha-1)}(a), U_{r+\alpha}(u)=u^{(\alpha-1)}(b), \quad \alpha=1,2, \cdots, n .
$$

As a physical example, we note that in the theory of strength of materials the deflection of a built-in beam is governed by a self-adjoint equation of the fourth order whose boundary conditions are

$$
u(a)=u^{\prime}(a)=u(b)=u^{\prime}(b)=0 .
$$

We shall now further assume that the left-hand end point (a) is held fixed while the right-hand end point (b) is allowed to vary. It follows from Theorem 3 of [MS] that if $L$ is self-adjoint, then 


$$
\delta G(x, \xi)=(-1)^{r} \frac{\partial^{r}}{\partial 2^{r}} G(\xi, b) \frac{\partial^{r}}{\partial 2^{r}} G(x, b) p_{0}(b) \delta b .
$$

TheOREM 1. Hypothesis. Let the interval $[a, b]$ be divided into $m+1$ subintervals by the points of subdivision $\zeta_{i}$ : $a<\zeta_{1}<\zeta_{2}<\cdots<\zeta_{m}<b$. Let $a_{i}, i=1,2, \cdots, m$, be any set of $m$ real numbers. Let $G\left(x, \xi \mid \zeta_{i}\right)$ be the Green's function for a self-adjoint differential operator of even order $n=2 r$ with boundary conditions of the form (8) in the interval $\left[a, \zeta_{i}\right]$. Let the completely homogeneous system be incompatible for every subinterval $[a, c]$ of $[a, b]$.

Conclusion. If $r$ is even,

$$
\begin{aligned}
\sum_{i=1}^{m} & \sum_{j=1}^{m} G\left(\zeta_{i}, \zeta_{j} \mid b\right) a_{i} a_{j} \\
& \geqq \sum_{i=1}^{m} \sum_{j=1}^{m} G\left(\zeta_{i}, \zeta_{j} \mid \zeta_{m}\right) a_{i} a_{j} \\
& =\sum_{i=1}^{m-1} \sum_{j=1}^{m-1} G\left(\zeta_{i}, \zeta_{j} \mid \zeta_{m}\right) a_{i} a_{j} \geqq \sum_{i=1}^{m-1} \sum_{j=1}^{m-1} G\left(\zeta_{i}, \zeta_{j} \mid \zeta_{m-1}\right) a_{i} a_{j} \\
& \geqq \cdots \geqq \sum_{i=1}^{m-k} \sum_{j=1}^{m-k} G\left(\zeta_{i}, \zeta_{j} \mid \zeta_{m-k}\right) a_{i} a_{j}=\sum_{i=1}^{m-k-1} \sum_{j=1}^{m-k-1} G\left(\zeta_{i}, \zeta_{j} \mid \zeta_{m-k}\right) a_{i} a_{j} \\
& \geqq \cdots \geqq G\left(\zeta_{1}, \zeta_{1} \mid \zeta_{2}\right) a_{1}^{2} \geqq G\left(\zeta_{1}, \zeta_{1} \mid \zeta_{1}\right) a_{1}^{2}=0 .
\end{aligned}
$$

If $r$ is odd, the inequalities will be reversed.

Proof.

$$
\begin{aligned}
\delta\left[\sum_{i=1}^{m} \sum_{j=1}^{m} G\left(\zeta_{i}, \zeta_{j}\right) a_{i} a_{j}\right] & \\
= & (-1)^{r} p_{0}(b) \delta b \sum_{i=1}^{m} \sum_{j=1}^{m} \frac{\partial^{r}}{\partial 2^{r}} G\left(\zeta_{i}, b\right) \frac{\partial^{r}}{\partial 2^{r}} G\left(\zeta_{j}, b\right) a_{i} a_{j} \\
= & (-1)^{r} p_{0}(b)\left[\sum_{k=1}^{m} \frac{\partial^{r}}{\partial 2^{r}} G\left(\zeta_{k}, b\right) a_{k}\right]^{2} \delta b
\end{aligned}
$$

by Equation (9) from which the positive or negative monotony of

$$
\sum_{i=1}^{m} \sum_{j=1}^{m} G\left(\zeta_{i}, \zeta_{j}\right) a_{i} a_{j}
$$

follows depending on whether $r$ is even or odd.

With a theorem such as this we can prove many interesting 
(mostly well known) results ${ }^{2}$ with a few strokes of the pen. For example:

CoRollary 1. Hypothesis. Let $G(s, t)$ be the Green's function for a self-adjoint differential operator $L$ of even order $n=2 r$ on the closed interval $[a, b]$ with boundary conditions of the form (8) such that the completely homogeneous system is incompatible for every subinterval $[a, c]$ of $[a, b]$.

CoNCLusion. If $\phi$ is any real continuous function in $[a, b]$,

$$
\int_{a}^{b} \int_{a}^{b} G(s, t) \phi(s) \phi(t) d s d t \geqq 0
$$

if $r$ is even. The inequality is reversed if $r$ is odd.

Proof. The non-negative sums of Equation (10) may be regarded as Riemann sums approximating the integral of Equation (11).

Corollary 2. Hypothesis. As in Corollary 1.

CoNCLusion. If $y(x)$ satisfies equation (8), then

$$
\int_{a}^{b} y(x) L y d x \geqq 0
$$

if $r$ is even. The inequality is reversed if $r$ is odd.

Proof. If we write $L y=\phi(x)$, then $y(x)=\int_{a}^{b} G(x, \xi) \phi(\xi) d \xi$ by the definition of the Green's function. Applying Corollary 1 we see that

$$
\int_{a}^{b} y(x) \phi(x) d x=\int_{a}^{b} y(x) L y(x) d x \geqq 0
$$

if $r$ is even.

An interesting result is the following:

Corollary 3. Hypothesis. As in Corollary 1.

CONCLUSION.

$$
|G(x, \xi)| \leqq[G(x, x) G(\xi, \xi)]^{1 / 2} \leqq(1 / 2)[G(x, x)+G(\xi, \xi)] .
$$

Proof. Let $m=2$ in Equation (10) and apply Schwarz's Inequality.

The representation in Theorem 1 can be generalized to include derivatives of the Green's functions.

Theorem 2. Hypothesis. As in Theorem 1 where we now introduce

2 A systematic presentation of well known properties of the Green's function can be found in the book Kernel functions in mathematical physics by S. Bergman and M. M. Schiffer, Academic Press, 1952. 
the real arbitrary constants $A_{\alpha i}, \alpha=0,1, \cdots, p \leqq r-1, i=1,2, \cdots$, $m$.

Conclusion.

$$
\sum_{\alpha=0}^{p} \sum_{\beta=0}^{p} \sum_{i=1}^{m} \sum_{j=1}^{m} A_{\alpha i} A_{\beta j} \frac{\partial^{\alpha+\beta}}{\partial 1^{\alpha} \partial 2^{\beta}} G\left(\zeta_{i}, \zeta_{j}\right) \geqq 0
$$

if $r$ is even. The inequality is reversed if $r$ is odd.

Proof. Apply the $\delta$ operator to

$$
\sum_{\alpha=0}^{p} \sum_{\beta=0}^{p} A_{\alpha i} A_{\beta j} \partial^{\alpha+\beta} G\left(\zeta_{i}, \zeta_{j}\right) / \partial 1^{\alpha} \partial 2^{\beta}
$$

and sum over $i$ and $j$.

As in the case of Theorem 1 we can deduce various corollaries.

Corollary 4. Hypothesis. Let $a_{\alpha}, \alpha=0,1, \cdots, p \leqq r-1$, be any set of $p+1$ real numbers. Let $G(s, t)$ be the Green's function for a selfadjoint linear differential operator $L$ of even order $n=2 r$ on the closed finite interval $[a, b]$ with boundary conditions of the form (8) such that the completely homogeneous system is incompatible in every subinterval $[a, c]$ of $[a, b]$.

CoNCLUSION. If $\phi$ is any real continuous function in $[a, b]$,

$$
\sum_{\alpha=0}^{p} \sum_{\beta=0}^{p} a_{\alpha} a_{\beta} \int_{a}^{b} \int_{a}^{b} \frac{\partial^{\alpha+\beta}}{\partial 1^{\alpha} \partial 2^{\beta}} G(s, t) \phi(s) \phi(t) d s d t \geqq 0
$$

if $r$ is even. The inequality is reversed if $r$ is odd.

Corollary 5. Hypothesis. As in Corollary 4. Conclusion.

$$
\begin{aligned}
& {\left[\int_{a}^{b} \int_{a}^{b} G_{1}(s, t) \phi(s) \phi(t) d s d t\right]^{2}} \\
& \quad \leqq\left[\int_{a}^{b} \int_{a}^{b} G(s, t) \phi(s) \phi(t) d s d t\right]\left[\int_{a}^{b} \int_{a}^{b} G_{11}(s, t) \phi(s) \phi(t) d s d t\right] .
\end{aligned}
$$

Proof. Let $p=1$ in Corollary 4 and apply Schwarz's Inequality.

We conclude this section with a theorem which has an obvious physical interpretation.

Theorem 3. Hypothesis. Let $G(x, \xi)$ be the Green's function for a self-adjoint linear differential operator of even order $n=2 r$ with boundary conditions of the form (8). Let $y(x)$ satisfy the differential equation $L y=\phi(x)$ and boundary conditions of the form (8). 
Conclusion.

$$
\frac{\delta y^{(r)}(b)}{y^{(r)}(b)}=(-1)^{r} p_{0}(b) \frac{\partial^{n}}{\partial x^{r} \partial \xi^{r}} G(b, b) \delta b .
$$

Proof. From $y(x)=\int_{b}^{a} G(x, \xi) \phi(\xi) d \xi$,

$$
\begin{aligned}
\delta y(x) & =(-1)^{r} \int_{a}^{b} \frac{\partial^{r}}{\partial 2^{r}} G(x, b) \frac{\partial^{r}}{\partial 2^{r}} G(\xi, b) p_{0}(b) \delta b \phi(\xi) d \xi \\
& =(-1)^{r} p_{0}(b) \delta b \frac{\partial^{r}}{\partial 2^{r}} G(x, b) \int_{a}^{b} \frac{\partial^{r}}{\partial 2^{r}} G(\xi, b) \phi(\xi) d \xi \\
& =(-1)^{r} p_{0}(b) \delta b \frac{\partial^{r}}{\partial 2^{r}} G(x, b) y^{(r)}(b) .
\end{aligned}
$$

[The fact that the upper limit of integration is $b$ does not affect the variation since the Green's function satisfies boundary conditions of the form (8).] Differentiate the above expression $r$ times with respect to $x$ and let $x=b$.

If we think of a loaded built-in beam whose deflection satisfies a self-adjoint equation of fourth order and self-adjoint boundary conditions of the form (8), we see that by measuring the rate of change of the first nonvanishing derivative $\left[y^{\prime \prime}(x)\right.$-the moment] of the deflection, $y(x)$, at the point $b$ we can obtain $\partial^{r} G(b, b) / \partial x^{r} \partial \xi^{r}$.

\subsection{Variation of the boundary conditions.}

THEOREM 4. HyPOTHESIS. Let $L$ be a self-adjoint differential operator of even order $n=2 r$ with boundary conditions of the form (8) in the interval $[a, b]$. Let the perturbed boundary conditions $U_{\alpha}^{\prime}$ be $U_{\alpha}^{\prime}=U_{\alpha}$ for $\alpha=1,2, \cdots, r-1, r+1, \cdots, 2 r-1$ and let $U_{r}^{\prime}(u)=\left(1+\epsilon_{1}\right) u^{(r-1)}(a)$ $+\eta_{1} u^{(r)}(a), U_{2 r}^{\prime}(u)=\left(1+\epsilon_{2}\right) u^{(r-1)}(b)+\eta_{2} u^{(r)}(b)$.

CONCLUSION.

$$
\begin{aligned}
\delta G(x, \xi)=(-1)^{r}\left\{\eta_{2} \frac{\partial^{r}}{\partial 2^{r}} G(x, b) \frac{\partial^{r}}{\partial 1^{r}} G(b, \xi) p_{0}(b)\right. & \\
& \left.-\eta_{1} \frac{\partial^{r}}{\partial 2^{r}} G(x, a) \frac{\partial^{r}}{\partial 1^{r}} G(a, \xi) p_{0}(a)\right\}
\end{aligned}
$$

Proof. From Theorem 5 of [MS],

$$
\Delta G(x, \xi)=\sum_{i=1}^{n} \varsigma \Delta U_{i}\left[G^{\prime}(\zeta, \xi)\right]^{\zeta} U_{2 n+1-i}^{*}[G(x, \zeta)] .
$$

We note that $\Delta U_{i}=0$ for $i=1,2, \cdots, r-1, r+1, \cdots, 2 r-1$ 
and from the expression for $\pi[u, v]$,

$$
\begin{aligned}
& { }^{\zeta} U_{3 r+1}^{*}[G(x, \zeta)]=-(-1)^{r} p_{0}(a) \frac{\partial^{r}}{\partial 2^{r}} G(x, a), \\
& { }^{\zeta} U_{2 r+1}^{*}[G(x, \zeta)]=(-1)^{r} p_{0}(b) \frac{\partial^{r}}{\partial 2^{r}} G(x, b),
\end{aligned}
$$

Substituting these formulae in $\Delta G(x, \xi)$ and making simplifications due to first order variation, we obtain Theorem 4.

We shall now define an "energy integral," $E[\phi]$ and show that it is monotonic. This theorem has a physical interpretation which we shall point out at the end of the proof.

TheOREM 5. Hypothesis. As in Theorem 4. Let $y(x)$ be any function which satisfies the boundary conditions $U_{\alpha}(y)=0, \alpha=1,2, \cdots, n$. Let $L y=\phi(x)$. Let $E[\phi]=\int_{a}^{b} \int_{a}^{b} G(x, \xi) \phi(x) \phi(\xi) d x d \xi$ by definition, where $G(x, \xi)$ is the Green's function.

Conclusion.

$$
\delta E=(-1)^{r}\left[\eta_{2} \overline{y^{(r)}(b)^{2}} p_{0}(b)-\eta_{1} \overline{y^{(r)}(a)^{2}} p_{0}(a)\right] .
$$

ProOF.

$$
\begin{aligned}
\delta E= & (-1)^{r} \eta_{2} p_{0}(b) \int_{a}^{b} \int_{a}^{b} \frac{\partial^{r}}{\partial 2^{r}} G(x, b) \frac{\partial^{r}}{\partial 1^{r}} G(b, \xi) \phi(x) \phi(\xi) d x d \xi \\
& -(-1)^{r} \eta_{1} p_{0}(a) \int_{a}^{b} \int_{a}^{b} \frac{\partial^{r}}{\partial 2^{r}} G(x, a) \frac{\partial^{r}}{\partial 1^{r}} G(a, \xi) \phi(x) \phi(\xi) d x d \xi .
\end{aligned}
$$

Noting that $y(\xi)=\int_{a}^{b} G(x, \xi) \phi(x) d x$, we easily arrive at the result stated in the theorem.

Physically speaking, if our self-adjoint differential operator represents the deflection of a beam, $L y=\phi$, then $y$ is the deflection and $\phi$ is the load. The original boundary conditions $U_{\alpha}(u), \alpha=1,2$, represent a built-in beam, while the $U_{\alpha}^{\prime}(u)$ represent conditions intermediate between a simply supported beam and a built-in beam. If the load is fixed, the energy varies monotonously as the boundary conditions vary from "built-in" to "simply supported."

2.3. Extensions to non self-adjoint operators. In all the previous applications of monotony we have assumed self-adjointness of the differential operator. In Theorem 6 below we shall prove a result concerning the monotony of the "energy" which is valid for non selfadjoint operators (but with self-adjoint boundary conditions). 
Theorem 6. Hypothesis. Let $L$ be a linear differential operator of order $n$ and let $U_{\alpha}(u)=0, \alpha=1,2, \cdots, m$, be a set of self-adjoint boundary conditions. Let $y(x), z(x)$ be two functions of class $C^{n}$ such that $U_{i}(y)=0=U_{i}(z)$ and $U_{2 n+1-i}^{*}(y)=U_{2 n+1-i}^{*}(z), i=1,2, \cdots, n$. Let $L^{+} y=\phi, L z=\psi$. Define the generalized energy integral as

$$
\Gamma[\phi, \psi]=\int_{a}^{b} \int_{a}^{b} G(x, \xi) \phi(x) \psi(\xi) d x d \xi\left[=\int_{a}^{b} z L^{+} y d x=\int_{a}^{b} y L z d x\right] .
$$

Let the perturbed boundary conditions be $U_{i}^{\prime}=U_{i}+\epsilon_{i} U_{2 n+1-i}^{*}, i=1$, $2, \cdots, n, \epsilon_{i} \geqq 0$.

CoNCLUSION. $\delta \Gamma \geqq 0$.

Proof. From Theorem 5 of [MS],

$$
\begin{aligned}
\delta \Gamma & =\sum_{i=1}^{n} U_{2 n+1-i}^{*}\left[\int_{a}^{b} G(x, t) \phi(x) d x\right] \cdot \delta U_{i}\left[\int_{a}^{b} G(t, \xi) \psi(\xi) d \xi\right] \\
& =\sum_{i=1}^{n} U_{2 n+1-i}^{*}(y) \cdot \delta U_{i}(z)=\sum_{i=1}^{n} \epsilon_{i} U_{2 n+1-i}^{*}(y) U_{2 n+1-i}^{*}(z) .
\end{aligned}
$$

3. Eigenvalue problems. Let us consider the eigenvalue problem

$$
L v_{i}(x)+\lambda_{i} v_{i}(x)=0, \quad U_{\alpha}\left(v_{i}\right)=0, \quad \alpha=1,2, \cdots, n,
$$

connected with the differential systems treated so far. We may write this in the form of an integral equation

$$
v_{i}(x)+\lambda_{i} \int_{a}^{b} G(x, \xi) v_{i}(\xi) d \xi=0
$$

by the use of the Green's function $G$ of the system $L v=0, U_{\alpha}(v)=0$. We perform upon this system a variation of the types considered in [MS], that is, we change the coefficients of the operator, the end points of the interval, or the boundary conditions. In each case, the variation $\delta G(x, \xi)$ of the Green's function has been determined, and by means of Equation (13), the variations $\delta v_{i}$ and $\delta \lambda_{i}$ of the corresponding eigenfunctions and eigenvalues can be deduced therefrom. Suppose, for simplicity, that the basic interval $[a, b]$ is kept fixed and that $\lambda_{i}$ is nondegenerate; then we have

$$
\begin{aligned}
0=\delta v_{i}(x)-\frac{\delta \lambda_{i}}{\lambda_{i}} v_{i}(x) & +\lambda_{i} \int_{a}^{b} \delta G(x, \xi) v_{i}(\xi) d \xi \\
& +\lambda_{i} \int_{a}^{b} G(x, \xi) \delta v_{i}(\xi) d \xi
\end{aligned}
$$


This represents an inhomogeneous integral equation of the second kind for $\delta v_{i}$ which contains, however, the unknown parameter $\delta \lambda_{i}$. In order to determine the latter, we multiply the above equation by $v_{i}(x)$ and integrate over the basic interval $[a, b]$. We may assume without loss of generality that $v_{i}(x)$ has been normalized by the requirement

$$
\int_{a}^{b} v_{i}(x)^{2} d x=1
$$

We then obtain, in view of Equation (13),

$$
\frac{\delta \lambda_{i}}{\lambda_{i}^{2}}=\int_{a}^{b} \int_{a}^{b} \delta G(x, \xi) v_{i}(\xi) v_{i}(x) d \xi d x .
$$

Thus the known variation formulae for the Green's function permit a determination of the change of eigenvalues $\lambda_{i}$, and then by the integral equation

$$
\delta v_{i}(x)+\lambda_{i} \int_{a}^{b} G(x, \xi) \delta v_{i}(\xi) d \xi=\frac{\delta \lambda_{i}}{\lambda_{i}} v_{i}(x)-\lambda_{i} \int_{a}^{b} \delta G(x, \xi) v_{i}(\xi) d \xi
$$

lead to a determination of the variation $\delta v_{i}$ of the eigenfunctions. As in previous applications, we may easily derive from Equation (14), in the case of important special forms of the differential system, monotony properties for the eigenvalues.

NEW YoRK UNIVERSITY AND

STANFORD UNIVERSITY 